\title{
The fracture liaison service, a step forward not only in fracture reduction, but also in mortality reduction
}

\author{
W. F. Lems ${ }^{1}$ ·. P. van den Bergh ${ }^{2}$ ·P. P. M. M. Geusens ${ }^{3}$
}

Received: 12 January 2022 / Accepted: 8 February 2022 / Published online: 15 February 2022

(c) International Osteoporosis Foundation and National Osteoporosis Foundation 2022

In a large prospective study in hip fracture patients, 357 patients were enrolled before and 744 after implementation of a fracture liaison service (FLS) [1, 2]. Based on age, gender, and-physical status, the patients seemed to be comparable. FLS-patients followed a protocol, including DXA in patients below 65 years of age, laboratory testing for secondary osteoporosis, mobility testing, and physical therapy.

After introduction, 91\% followed the FLS-protocol, and in 78\% anti-osteoporotic drugs, according to the recommendations of the IOF [3], were initiated, versus in $12 \%$ of usual care patients. Fracture rate was reported after 2 years: $7.3 \%$ before and $6.6 \%$ after introduction of FLS (no significant difference). Mortality rate was lower in the first year after introduction of FLS than before: $19.8 \%$ versus $25.8 \%$; HR: $0.76(0.58-0.98)$, but not in the second year: $31.8 \%$ versus $29.8 \%$. The lower mortality rate in the first year could be related to interventions, such as fall risk interventions and stimulating a healthy lifestyle.

A lack of adherence to osteoporotic drugs could be a reason for the absence of a favorable effect on mortality in the second year. Only 3.3\% of patients before FLS and 30.5\% of patients after FLS-introduction were adherent to drug treatment, which is suboptimal, as in many other studies [4].

Mortality risk was observed in previous studies. In a 15-year observational study in 6120 participants, a $34 \%$

W. F. Lems

wf.lems@amsterdamumc.nl

1 Amsterdam University Medical Center, Amsterdam, the Netherlands

2 Department of Internal Medicine, Subdivison of Rheumatology, Maastricht University Medical Center, Maastricht, the Netherlands

3 Department of Medicine and Life Sciences, Hasselt University, Hasselt, Belgium mortality risk-reduction was reported in bisphosphonate users compared to controls [5]. In another study, comparing FLS attenders to non-attenders after 8 years of follow-up, with 5011 patients, a $40 \%$ lower subsequent fracture rate and a $21 \%$ lower mortality were found [6].

In a recent systematic literature review and meta-analysis, in nine before and after FLS implementation studies, it was found that FLS-care was associated with a significantly lower probability of fractures (HR 0.70; 95\% C.I.: $0.52-0.93$ ) and a lower probability of mortality (HR 0.65 ; 95\% C.I.: 0.44-0.95) [7].

It is important to realize that "before-after studies" are not the highest level of methodology but performing "placebocontrolled" studies in patients with a hip fracture at the FLS is regarded as unethical, since the study with zoledronic acid versus placebo in hip fracture patients, in which a reduction of vertebral and non-vertebral fractures and of mortality was found [8].

What can we learn from this study? First, that it is feasible to initiate an FLS for hip fracture patients, with favorable effects on the proportion of patients following the FLSprotocol and starting anti-osteoporotic treatment, while long-term adherence and monitoring are another critical issue. The authors questioned whether FLS could reduce mortality [1], but in larger studies, not only a reduction in fractures, but also in mortality has been found. High on the research agenda are further, long-term studies with competing mortality risk analysis to better understand the interaction between mortality rate and subsequent fracture risk [7].

Secondly, we need studies focusing on the optimal implementation of post-fracture care, including diagnosis, treatment and adherence and monitoring, as described in the key performance indicator (KPI) set of IOF Capture the Fracture [9]. All KPIs are important, and the chain is as strong as the weakest link. 


\section{Declarations}

Conflict of interest Prof Lems: speakers' fee and advisory boards: Amgen, UCB, Eli Lilly, Pfizer, Galapagos. Prof van den Bergh: research funding from UCB and Amgen, consultant for UCB and Amgen. Prof Geusens: lectures and participation in clinical trials: Abbott, Amgen, BMS, Celgene, Janssen, Lilly, MSD, Merck, Novartis, Pfizer, Roche, UCB, Will-Pharma, Fresenius, Viatris, Sandoz, Galapagos.

\section{References}

1. Index artikel: Gonzalez-Quevedo D, Perez del Rio V, Garcesco Moriel D, et al. A two-year follow-up of a novel fracture liaison service: can we reduce the mortality in elderly hip fracture patients? A prospective cohort study. Ost Int

2. Gonzalez-Quevedo D, Bautista Enrique D, Perez del Rio V et al (2020) Fracture liaison service and mortality in elderly hip fracture patients. Ost Int 31:77-84

3. Kanis JA, Cooper C et al (2019) European guidance for the diagnosis and treatment of osteoporosis in postmenopausal women. Ost Int 30:3-44
4. Imaz I, Zegarra P, Gonzalez J et al (2010) Poor bisphosphonate adherence for treatment of osteoporosis increases fracture risk: systematic review and meta-analysis. Osteoporos Int 21:1943-51

5. Bliuc D, Tran T, van Geel T et al (2019) Mortality risk reduction differs according to bisphosphonate class: a 15-year observational study. Ost Int 30:817-828

6. Van Geel TACM, Bliuc D, Geusens P, et al. (2018) Reduced mortality and subsequent fracture risk associated with oral bisphosphonate recommendation a fracture liaison service setting: a prospective cohort study. Plos One 13 (6)

7. Li N, Hilligsman M, Boonen A et al (2021) The impact of the fracture liaison service on subsequent fractures and mortality: a systematic literature review and meta-analysis. Ost Int 32(8): 1517-1530

8. Lyles KW, Colon-Emeric CS, Magaziner JS et al (2007) Zoledronic acid and clinical fractures and mortality after hip fractures. New Engl J Med 357:1799-1809

9. Javaid MK, Sami A, Lems W et al (2020) A patient-level key performance indicator set to measure the effectiveness of FLS and guide quality improvement: a position paper of the IOF Capture the Fracture. Ost Int 31:1193-1204

Publisher's note Springer Nature remains neutral with regard to jurisdictional claims in published maps and institutional affiliations. 